\title{
Successful single-session cricopharyngeal and Zenker's diverticulum peroral endoscopic myotomy
}

Therapy of symptomatic Zenker's diverticulum can be accomplished with open surgery, rigid endoscopy, or flexible endoscopy [1]. Peroral endoscopic myotomy (POEM) can be used to treat Zenker's diverticulum using the principles of submucosal tunneling. Zenker's POEM has potential advantages over traditional endoscopic septotomy, including complete septum division and mucosal integrity during septotomy. Importantly, Zenker's POEM may be associated with a decreased risk of symptom recurrence, because of the complete exposure and dissection of the septum ( $\triangleright$ Table 1 ). We describe a patient with a hypertensive

\begin{tabular}{|c|c|c|}
\hline & Endoscopic septotomy & POEM \\
\hline Complete septum division & $+1-$ & ++ \\
\hline Mucosal integrity during septotomy & - & ++ \\
\hline Ease of perforation closure & $+1-$ & ++ \\
\hline Risk of symptom recurrence & ++ & - \\
\hline
\end{tabular}

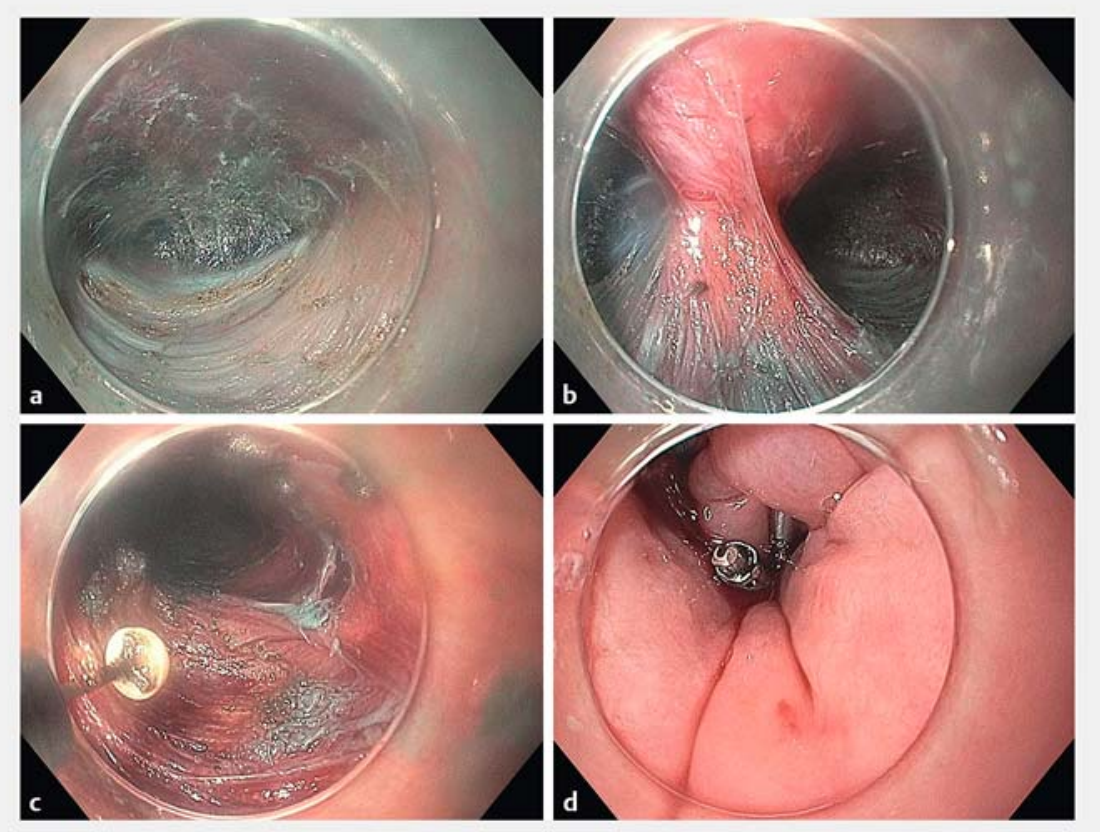

- Fig. 1 The peroral endoscopic myotomy (POEM) technique for Zenker's diverticulotomy. a A submucosal tunnel was created using spray coagulation and injection of saline with indigo carmine solution via the pump. b The finished tunnel shows complete exposure of the septum. c Zenker's diverticulum septotomy and cricopharyngeus muscle myotomy were performed using endoscopic submucosal dissection knives. $\mathbf{d}$ The mucosal incision was securely closed using six through-the-scope clips.

cricopharyngeus muscle and Zenker's diverticulum successfully treated with POEM in the same session.

A 94-year-old woman with a past medical history of hypertension and atrial fibrillation was referred with daily symptoms of solid and liquid food dysphagia and regurgitation, which occurred with every meal. She denied weight loss and dyspnea. A barium esophagogram revealed a 4-cm Zenker's diverticulum and the decision was made to perform Zenker's POEM.

A diagnostic gastroscope fitted with a clear cap was advanced. A very tight cricopharyngeus muscle was noted at $17 \mathrm{~cm}$ from the incisors and a large Zenker's diverticulum was identified. A mucosal bleb was created $2 \mathrm{~cm}$ above the cricopharyngeus muscle at $15 \mathrm{~cm}$ from the incisors. A $1.5-\mathrm{cm}$ incision was made with a triangle-tip knife using a predetermined electrocautery setting. The submucosal fibers were dissected with spray coagulation and the endoscope was advanced to the submucosal space. A submucosal tunnel was created using spray coagulation and injection of saline with indigo carmine solution via the pump ( $\triangleright$ Fig. 1 a). When vessels were identified they were treated using a coagulation grasper with soft coagulation. The septum of the Zenker's diverticulum was identified.

After the tunnel on the esophageal side of the septum had been completed, the tunnel on the diverticular side was commenced and completed with complete exposure of the septum ( $\triangleright$ Fig. 1 b). Septotomy was then performed using a combination of the insulated-tip knife and the triangle-tip knife with spray coagulation current. After the septotomy had been completed, the tight cricopharyngeus muscle was identified and cricopharyngeal myotomy was performed using the insulated-tip knife in retrograde fashion ( $\mathbf{F i g . 1} \mathbf{c}$ ). This resulted in easy passage of the endoscope 


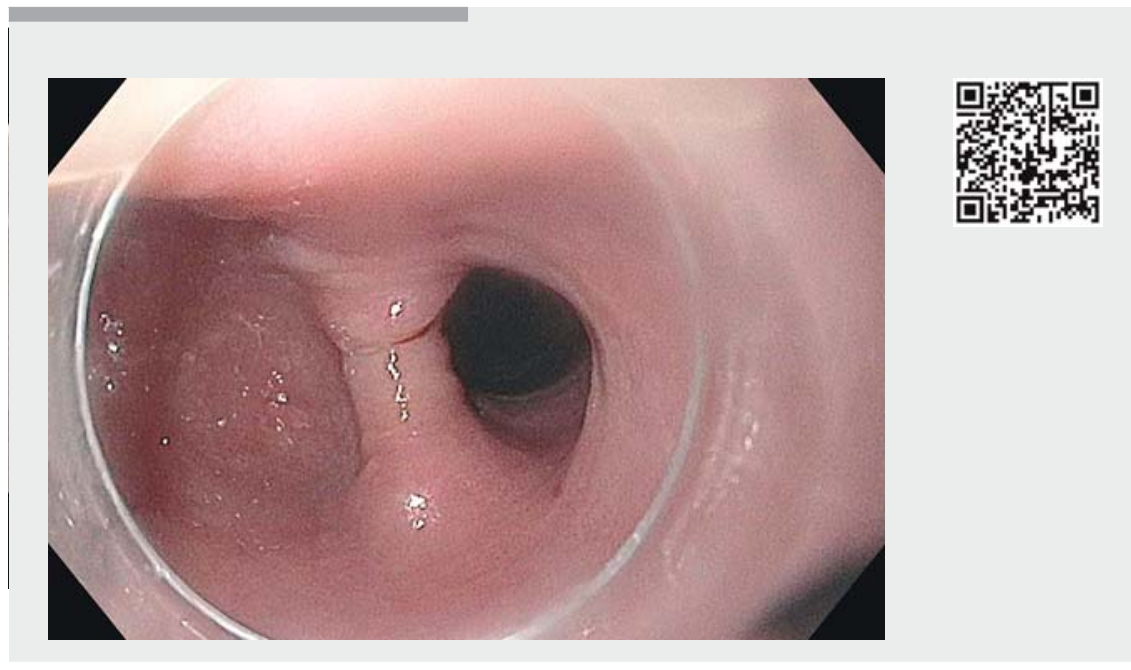

$\checkmark$ Video 1 A 94-year-old woman with solid and liquid food dysphagia and regurgitation is treated by peroral endoscopic myotomy for both a tight cricopharyngeus and a Zenker's diverticulum in a single session.

across the upper esophageal sphincter. The mucosal incision was securely closed using six through-the-scope clips ( $\bullet$ Fig. 1 d; : Video 1).

There were no complications and the patient was admitted overnight. An esophagogram performed the next day showed no leakage. At follow-up 3 months postPOEM, she had compete resolution of her symptoms with no residual dysphagia or regurgitation.

Zenker's diverticulotomy using the POEM technique allows complete exposure and dissection of the septum, which potentially may eliminate the risk of recurrence. Furthermore, we have demonstrated, for the first time, the use of POEM to intervene on a tight upper esophageal sphincter in the same session.

Endoscopy_UCTN_Code_TTT_1AO_2AD
Mouen A. Khashab, MD

Johns Hopkins Hospital, Sheikh Zayed Bldg, Division of Gastroenterology and Hepatology, 1800 Orleans Street, Suite 7125G, Baltimore, MD 21287, USA

Fax: +1-443-683-8335

mkhasha1@jhmi.edu

\section{Reference}

[1] Li QL, Chen WF, Zhang XC. Submucosal tunneling endoscopic septum division: a novel technique for treating Zenker's diverticulum. Gastroenterology 2016; 151: 1071 1074

\section{Bibliography}

DOI https://doi.org/10.1055/a-0624-1977

Published online: 12.6.2018

Endoscopy 2018; 50: E220-E221

(c) Georg Thieme Verlag KG

Stuttgart - New York

ISSN 0013-726X

Anthony N. Kalloo is a founding member and Equity Holder of Apollo Endosurgery. Vivek Kumbhari is a consultant for Apollo Endosurgery, ReShape Life Sciences, Medtronic, and Boston Scientific. Mouen A. Khashab is a consultant and on the medical advisory boards for Boston Scientific and Olympus. The remaining authors have no disclosures.

The authors

Olaya I. Brewer Gutierrez, Robert Moran, Juliana Yang, Omid Sanaei, Anthony N. Kalloo, Vivek Kumbhari, Mouen A. Khashab Division of Gastroenterology and Hepatology, Johns Hopkins Hospital, Baltimore, Maryland, USA

\section{ENDOSCOPY E-VIDEOS}

https://eref.thieme.de/e-videos

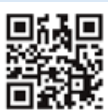

Endoscopy E-Videos is a free access online section, reporting on interesting cases and new techniques in gastroenterological endoscopy. All papers include a high quality video and all contributions are freely accessible online.

This section has its own submission website at https://mc.manuscriptcentral.com/e-videos 